\title{
On Bar Frameworks, Stress Matrices and Semidefinite Programming
}

\author{
A. Y. Alfakih *广 \\ Department of Mathematics and Statistics \\ University of Windsor \\ Windsor, Ontario N9B 3P4 \\ Canada
}

August 6, 2009

AMS classification: 90C22, 52C25, 05C62, 15A57

Keywords: Semidefinite programming, bar frameworks, universal rigidity, stress matrices, Gale transform.

\begin{abstract}
A bar framework $G(p)$ in $r$-dimensional Euclidean space is a graph $G=$ $(V, E)$ on the vertices $1,2, \ldots, n$, where each vertex $i$ is located at point $p^{i}$ in $\mathbb{R}^{r}$. Given a framework $G(p)$ in $\mathbb{R}^{r}$, a problem of great interest is that of determining whether or not there exists another framework $G(q)$, not obtained from $G(p)$ by a rigid motion, such that $\left\|q^{i}-q^{j}\right\|^{2}=\left\|p^{i}-p^{j}\right\|^{2}$ for all $(i, j) \in E$. This problem is known as either the global rigidity problem or the universal rigidity problem depending on whether such a framework $G(q)$ is restricted to be in the same $r$-dimensional space or not. The stress matrix $S$ of a bar framework $G(p)$ plays a key role in these and other related problems.

In this paper, we show that semidefinite programming (SDP) can be effectively used to address the universal rigidity problem. In particular, we use the notion of non-degeneracy of SDP to obtain a sufficient condition for universal rigidity, and to re-derive the known sufficient condition for generic universal rigidity. We present new results concerning positive semidefinite stress matrices and we use a semidefinite version of Farkas lemma to characterize bar frameworks that admit a nonzero positive semidefinite stress matrix $S$.
\end{abstract}

${ }^{*}$ E-mail: alfakih@uwindsor.ca

${ }^{\dagger}$ Research supported by the Natural Sciences and Engineering Research Council of Canada. 


\section{Introduction}

A configuration $p$ in $r$-dimensional Euclidean space is a finite collection of points $p^{1}, \ldots, p^{n}$ in $\mathbb{R}^{r}$ that affinely span $\mathbb{R}^{r}$. A bar framework in $\mathbb{R}^{r}$ (or a framework ${ }^{1}$ for short), denoted by $G(p)$, is a configuration $p$ in $\mathbb{R}^{r}$ together with a simple graph $G=(V, E)$ on the vertices $1,2, \ldots, n$, where each vertex $i$ of $G$ is located at $p^{i}$. Figure 1 depicts two frameworks in the plane. The vertices of $G$ are represented by little circles, while the edges of $G$ are represented as line-segments (or bars). With a slight abuse of notation, we will, sometimes, refer to the vertices and edges of $G$ as the vertices and the edges of the framework $G(p)$.

Two frameworks $G(p)$ and $G(q)$ in $\mathbb{R}^{r}$ are said to be congruent if $\left\|q^{i}-q^{j}\right\|=$ $\left\|p^{i}-p^{j}\right\|$ for all $i, j=1, \ldots, n$, where $\|$.$\| denotes the Euclidean norm. That is, G(p)$ and $G(q)$ are congruent if configuration $q$ can be obtained from configuration $p$ by applying a rigid motion such as a translation or a rotation in $\mathbb{R}^{r}$. On the other hand, two frameworks $G(p)$ in $\mathbb{R}^{r}$ and $G(q)$ in $\mathbb{R}^{s}$ are said to be equivalent if $\left\|q^{i}-q^{j}\right\|=$ $\left\|p^{i}-p^{j}\right\|$ for all $(i, j) \in E$. The term "bar" is used to denote such frameworks since in any two equivalent frameworks, every two adjacent vertices of $G(p)$ stay the same distance apart. Thus one can think of each edge of $G(p)$ as a stiff bar.

A framework $G(p)$ in $\mathbb{R}^{r}$ is said to be globally rigid if there does not exist a framework $G(q)$ in the same $r$-dimensional Euclidean space that is equivalent, but not congruent, to $G(p)$. Furthermore, if there does not exist a framework $G(q)$ in any Euclidean space that is equivalent, but not congruent, to $G(p)$, then $G(p)$ is said to be universally rigid ${ }^{2}$. Obviously, universal rigidity implies global rigidity, however, the converse need not be true. Framework (b) in Figure 1 is globally rigid but not universally rigid, since it has no equivalent, non-congruent, framework in $\mathbb{R}^{2}$, and since it has an infinite number of equivalent, non-congruent, frameworks in $\mathbb{R}^{3}$ and $\mathbb{R}^{4}$.

The global and universal rigidity problems of frameworks can also be posed in the context of the graph realization problem (GRP). Given an edge-weighted graph $G=(V, E, \omega)$ where $\omega_{i j}$ represents a positive weight on the edge $(i, j)$. A realization of $G$ in $\mathbb{R}^{r}$ is a mapping of the vertices $1,2, \ldots, n$ of $G$ into points $p^{1}, p^{2}, \ldots, p^{n}$ in $\mathbb{R}^{r}$ such that $\left\|p^{i}-p^{j}\right\|^{2}=\omega_{i j}$ for each $(i, j) \in E$. The GRP is the problem of determining whether or not $G$ has a realization in $\mathbb{R}^{r}$. Thus, the problems of global rigidity and universal rigidity can be stated as the problems of determining whether or not, a given realization of $G$ in $\mathbb{R}^{r}$ is unique, up to a rigid motion, in $\mathbb{R}^{r}$ or in all Euclidean spaces.

\footnotetext{
${ }^{1}$ Only bar frameworks are considered here. Tensegrity frameworks fall outside the scope of this paper.

${ }^{2}$ There are many other notions of rigidity such as rigidity, infinitesimal rigidity, dimensional rigidity etc. However, these notions will not be discussed in this paper.
} 


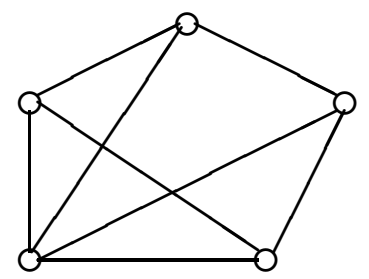

(a)

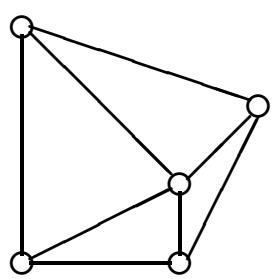

(b)

Figure 1: Two bar frameworks in $\mathbb{R}^{2}$. Framework (a) is universally rigid while framework (b) is globally rigid but not universally rigid.

The GRP and the global and universal rigidity of frameworks have important applications in molecular conformations [11], multidimensional scaling [16, 10] and wireless sensor networks $[22,13]$. In particular, the wireless sensor network localization problem is a special case of the GRP where $r=2$ or 3 , and where $G$ has a clique of size at $r+1$.

The GRP is well known to be NP-hard [20]. Semidefinite programming (SDP) was successfully used in [5] to solve a relaxation of the GRP by asking whether a given edge-weighted graph $G$ has a realization in some Euclidean space, not necessarily in a given $r$-dimensional space. SDP also was successfully used in $[7,12,22,18,23]$ to solve the wireless senor network localization problem, and in [21] to solve some problems in tensegrity theory. A tensegrity framework is a generalization of a bar framework where the edges of graph $G$ are labeled as bars, cables or struts. If an edge $(i, j)$ is labeled as a cable (strut), then $\left\|p^{i}-p^{j}\right\|$ is constrained to be $\leq(\geq)$ a certain given value.

The stress matrix $S$ of a framework $G(p)$ is closely related to the Gale matrix of $G(p)$. It plays a key role in the characterization of generic global rigidity and in establishing the universal rigidity of a given generic framework $G(p)^{3}$.

In this paper, we show that SDP can be effectively used to address the universal rigidity problem. In particular, we use the notion of SDP non-degeneracy to obtain a sufficient condition for universal rigidity, and to re-derive the known sufficient condition for generic universal rigidity. We present new results concerning positive semidefinite stress matrices and we use a semidefinite version of the Farkas lemma to characterize frameworks that admit a nonzero positive semidefinite stress matrix $S$.

We denote by $\mathcal{S}_{n}$ the subspace of symmetric matrices of order $n$. The positive semi-definiteness (definiteness) of a symmetric matrix $A$ is denoted by $A \succeq 0(A \succ$ $0)$. $e$ denotes the vector of all ones in $\mathbb{R}^{n}$, and $I_{n}$ denotes the identity matrix of order $n$. $E^{i j}$ denotes the $n \times n$ symmetric matrix with 1 's in the $(i, j)$ th and $(j, i)$ th entries and zeros elsewhere.

\footnotetext{
${ }^{3}$ Stress matrices even play a more important role in tensegrity theory.
} 


\section{Preliminaries}

\subsection{The Stress Matrix $S$ and the Gale Matrix $Z$}

An equilibrium stress of a framework $G(p)$ is a real valued function $\omega$ on $E$, the set of edges of $G$, such that

$$
\sum_{j:(i, j) \in E} \omega_{i j}\left(p^{i}-p^{j}\right)=0 \text { for all } i=1, \ldots, n \text {. }
$$

One may think of $\omega_{i j}\left(p^{i}-p^{j}\right)$ as the force exerted by the edge (bar) $(i, j)$ on node $i$. This force is called a tension in the bar $(i, j)$ if $\omega_{i j}<0$, and it is called a compression in the bar $(i, j)$ if $\omega_{i j}>0$. Thus equation (1) is equivalent to the statement that the net force acting on each node $i$ is equal to zero.

Let $\omega$ be an equilibrium stress of $G(p)$. Then the $n \times n$ symmetric matrix $S=\left(s_{i j}\right)$ where

$$
s_{i j}= \begin{cases}-\omega_{i j} & \text { if }(i, j) \in E, \\ 0 & \text { if }(i, j) \notin E, \\ \sum_{k:(i, k) \in E} \omega_{i k} & \text { if } i=j,\end{cases}
$$

is called the stress matrix associated with $\omega$, or a stress matrix of $G(p)$.

Let $G(p)$ be a framework with $n$ vertices in $\mathbb{R}^{r}$. Then it immediately follows that the following $(r+1) \times n$ matrix

$$
A:=\left[\begin{array}{cccc}
p^{1} & p^{2} & \ldots & p^{n} \\
1 & 1 & \ldots & 1
\end{array}\right]
$$

has full row rank since $p^{1}, \ldots, p^{n}$ affinely span $\mathbb{R}^{r}$. Note that $r \leq n-1$. Let $\bar{r}$ be the dimension of the nullspace of $A$; i.e., $\bar{r}=n-1-r$. For $\bar{r} \geq 1$, let $\Lambda$ be the $n \times \bar{r}$ matrix whose columns form a basis for the nullspace of $A$. $\Lambda$ is called a Gale matrix corresponding to $G(p)$. We will exploit the fact that $\Lambda$ is not unique to define a special sparse Gale matrix $Z$ which is more convenient for our purposes.

Let us write $\Lambda$ in block form as

$$
\Lambda=\left[\begin{array}{l}
\Lambda_{1} \\
\Lambda_{2}
\end{array}\right]
$$

where $\Lambda_{1}$ is $\bar{r} \times \bar{r}$ and $\Lambda_{2}$ is $(r+1) \times \bar{r}$. Since $\Lambda$ has full column rank, we can assume without loss of generality that $\Lambda_{1}$ is nonsingular. Then $Z$ is defined by

$$
Z:=\Lambda \Lambda_{1}^{-1}=\left[\begin{array}{c}
I_{\bar{r}} \\
\Lambda_{2} \Lambda_{1}^{-1}
\end{array}\right] .
$$




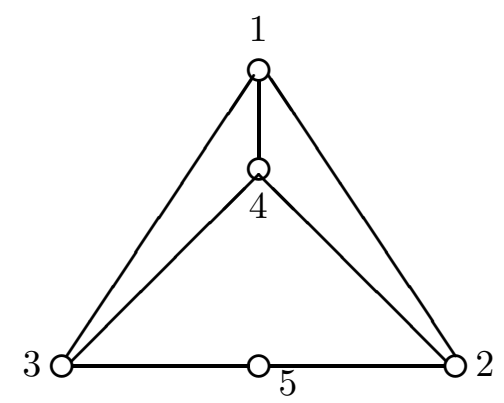

Figure 2: The non-generic framework in $\mathbb{R}^{2}$ of Example 2.1.

$Z$ is called the Gale matrix of $G(p)$. Furthermore, let $z^{i^{T}}$ denote the $i$ th row of $Z$. Then the $\bar{r}$-vector $z^{i}$ is called the Gale transform of $p^{i}[14]$.

The following theorem shows that the Gale matrix $Z$ of framework $G(p)$ is closely related to the stress matrix $S$ associated with an equilibrium stress $\omega$ of $G(p)$.

Lemma 2.1 (Alfakih [4]) Given a framework $G(p)$ with $n$ vertices in $\mathbb{R}^{r}$, let $Z$ be the Gale matrix of $G(p)$ and recall that $\bar{r}=n-1-r$. Further, let $S$ be a stress matrix of $G(p)$. Then there exists an $\bar{r} \times \bar{r}$ symmetric matrix $\Psi$ such that

$$
S=Z \Psi Z^{T}
$$

On the other hand, let $\Psi^{\prime}$ be any $\bar{r} \times \bar{r}$ symmetric matrix such that $z^{i T} \Psi^{\prime} z^{j}=0$ for all $(i, j) \notin E$, where $z^{i^{T}}$ denotes the ith row of $Z$. Then $S^{\prime}=Z \Psi^{\prime} Z^{T}$ is a stress matrix of $G(p)$.

Example 2.1 Consider the framework $G(p)$ in Figure 2, where $p^{1}=\left[\begin{array}{ll}0 & 2\end{array}\right]^{T}, p^{2}=$ $\left[\begin{array}{ll}2 & -1\end{array}\right]^{T}, p^{3}=\left[\begin{array}{ll}-2 & -1\end{array}\right]^{T}, p^{4}=\left[\begin{array}{ll}0 & 1\end{array}\right]^{T}$ and $p^{5}=\left[\begin{array}{ll}0 & -1\end{array}\right]^{T}$. Its Gale matrix is:

$$
Z=\left[\begin{array}{rr}
1 & 0 \\
0 & 1 \\
0 & 1 \\
-3 / 2 & 0 \\
1 / 2 & -2
\end{array}\right]
$$

It is easy to show that $G(p)$ has an equilibrium stress $\omega=\left(\omega_{12}=-1, \omega_{13}=\right.$ $\left.-1, \omega_{14}=6, \omega_{24}=3 / 2, \omega_{25}=-1 / 2, \omega_{34}=3 / 2, \omega_{35}=-1 / 2\right)$, and a stress matrix

$$
S=\left[\begin{array}{rrrrr}
4 & 1 & 1 & -6 & 0 \\
1 & 0 & 0 & -3 / 2 & 1 / 2 \\
1 & 0 & 0 & -3 / 2 & 1 / 2 \\
-6 & -3 / 2 & -3 / 2 & 9 & 0 \\
0 & 1 / 2 & 1 / 2 & 0 & -1
\end{array}\right]=Z \Psi Z^{T}
$$

where $\Psi=\left[\begin{array}{ll}4 & 1 \\ 1 & 0\end{array}\right]$. 


\subsection{Generic Frameworks}

The problems of framework global rigidity and universal rigidity become easier if we consider frameworks with a "typical" or generic configuration. A configuration $p$ (or a framework $G(p))$ in $\mathbb{R}^{r}$ is said to be generic if all the coordinates of $p^{1}, \ldots, p^{n}$ are

algebraically independent over the integers. That is, $G(p)$ is generic if there does not exist a non-zero polynomial $f$ with integer coefficients such that $f\left(p^{1}, \ldots, p^{n}\right)=0$.

The notion of generic frameworks can be weakened to that of frameworks in general position. A framework $G(p)$ in $\mathbb{R}^{r}$ is said to be in general position if no subset of the points $p^{1}, \ldots, p^{n}$ of cardinality $r+1$ is affinely dependent. For example, a set of points in the plane are in general position if no 3 of them lie on a straight line.

The following lemma will be needed in Section 6. For a proof see [3].

Lemma 2.2 Let $G(p)$ be a framework in general position with $n$ vertices in $\mathbb{R}^{r}$ and let $z^{1}, \ldots, z^{n}$ be the Gale transform of $p^{1}, \ldots, p^{n}$ respectively. Recall that $\bar{r}=n-1-r$. Then any subset of $z^{1}, \ldots, z^{n}$ of cardinality $\bar{r}$ is linearly independent.

The following are two recently obtained results concerning generic framework global and universal rigidity.

Theorem 2.1 (Connelly [9] Gortler et al [15]) Let $G(p)$ be a generic framework of $n$ vertices in $\mathbb{R}^{r}$. Then $G(p)$ is globally rigid if and only if:

$$
\exists \text { a stress matrix } S \text { of } G(p) \text { such that rank } S=\bar{r}=n-1-r \text {. }
$$

Theorem 2.2 (Alfakih [4], Connelly [8]) Let $G(p)$ be a generic framework of $n$ vertices in $\mathbb{R}^{r}$. Then $G(p)$ is universally rigid if

$\exists$ a stress matrix $S$ of $G(p)$ such that $S \succeq 0$ and rank $S=\bar{r}=n-1-r$.

Note that in light of Lemma 2.1, Conditions (5) and (6) are equivalent, respectively, to the two following conditions, where $z^{1}, z^{2}, \ldots, z^{n}$ are the Gale transforms of $p^{1}, p^{2}, \ldots, p^{n}$.

$$
\begin{aligned}
& \exists \bar{r} \times \bar{r} \text { nonsingular symmetric matrix } \Psi: z^{i^{T}} \Psi z^{j}=0, \forall(i, j) \notin E, \\
& \exists \bar{r} \times \bar{r} \text { matrix } \Psi \succ 0 \text { such that } z^{i^{T}} \Psi z^{j}=0, \forall(i, j) \notin E .
\end{aligned}
$$

Also, note that the assumption in Theorems 2.1 and 2.2 that the framework is generic can not be dropped. The framework of Example 2.1 depicted in Figure 2, which is clearly non-generic, is not globally rigid even though it satisfies Condition (5). Similarly, the non-generic framework of Example 6.1 is not universally rigid even though it satisfies Condition (6). 


\section{A Characterization of Equivalent Frameworks}

The first step in making the universal rigidity problem amenable to semidefinite programming is to use a Gram matrix, or more accurately a projected Gram matrix, to represent a configuration $p$. This enables us to characterize the set of all frameworks that are equivalent to a given framework $G(p)$.

Given a configuration $p=\left(p^{1}, \ldots, p^{n}\right)$ in $\mathbb{R}^{r}$, the $n \times r$ matrix

$$
P:=\left[\begin{array}{c}
p^{1^{T}} \\
p^{2^{T}} \\
\vdots \\
p^{n T}
\end{array}\right]
$$

is called the configuration matrix of $p$. The $n \times n$ symmetric matrix $B:=P P^{T}$ is called the Gram matrix associated with configuration $p$.

Note that in addition to being positive semidefinite of rank $r$, matrix $B$ is invariant under orthogonal transformations. Furthermore, wlog we assume that in any configuration $p$, the origin coincides with the centroid of the points $p^{1}, \ldots, p^{n}$; i.e., $B e=0$. Then, $B$ becomes also invariant under translations. Consequently, all congruent frameworks have the same Gram matrix. Hence, representing frameworks by Gram matrices, instead of configuration matrices, allows us to identify all congruent frameworks. Therefore, in the sequel we don't distinguish between congruent frameworks.

The set $\left\{C \in \mathcal{S}_{n}: C \succeq 0, C e=0\right\}$ forms a face of the cone of $n \times n$ symmetric positive semidefinite matrices. This face is isomorphic to the cone of positive semidefinite matrices of order $n-1$. For our purposes, it is more convenient to work in this latter cone. To this end, let $V$ be an $n \times(n-1)$ matrix such that

$$
V^{T} e=0, \quad V^{T} V=I_{n-1} .
$$

Definition 3.1 Given a configuration $p=\left(p^{1}, p^{2}, \ldots, p^{n}\right)$ in $\mathbb{R}^{r}$. The projected Gram matrix $X$ associated with $p$ is the $(n-1) \times(n-1)$ matrix

$$
X:=V^{T} B V,
$$

where $B$ is the Gram matrix associated with $p$.

Note that $X$ is an $(n-1) \times(n-1)$ positive semidefinite matrix of rank $r$.

Given a configuration $p$, the projected Gram matrix associated with $p$ can be easily computed using equation (11). On the other hand, given a projected Gram matrix $X$, configuration $p$ can be recovered as follows. Compute the Gram matrix 
$B$ which is given by $B=V X V^{T}$. Then factorize $B$ as $B=P P^{T}$. This can be done since $B$ is positive semidefinite. Furthermore, since rank $B=r$ and $B e=0$, it follows that $P$ is an $n \times r$ matrix such that $P^{T} e=0$. Thus the points $p^{1}, \ldots, p^{n}$ are simply given by the rows of $P$. Furthermore, their centroid coincides with the origin. Recall that we do not distinguish between congruent configurations. Thus, the configuration matrix $P$, the Gram matrix $B$ and the projected Gram matrix $X$ uniquely determine one another. Hence, the terms "framework $G(p)$ " and "framework $G(X)$ " can be used interchangeably. For more details see [3].

Let $G(\hat{p})$ be a given framework in $\mathbb{R}^{r}$. To avoid trivialities, we assume that $G=(V, E)$ is not the complete graph. Let $\bar{m}$ denote the number of missing edges of $G$. For each $(i, j) \notin E$ define the matrix

$$
M^{i j}:=\frac{1}{2} V^{T} E^{i j} V
$$

Recall that $E^{i j}$ is the $n \times n$ matrix with 1 's in the $(i, j)$ th and $(j, i)$ th entries and 0 's elsewhere. Let $\hat{X}$ be the projected Gram matrix corresponding to $G(\hat{p})$ and let

$$
\Omega:=\left\{y \in \mathbb{R}^{\bar{m}}: X(y):=\hat{X}+\sum_{(i, j) \notin E} y_{i j} M^{i j} \succeq 0\right\}
$$

and

$$
\Omega_{r}:=\{y \in \Omega: \operatorname{rank} X(y)=r\} .
$$

Note that the origin $(y=0)$ always belongs to $\Omega$ and to $\Omega_{r}$ since $\hat{X}$ is positive semidefinite of rank $r$. It was shown in [1] that the set of all frameworks $G(q)$ in $\mathbb{R}^{s}$, $1 \leq s \leq n-1$, that are equivalent to $G(\hat{p})$ is given by

$$
\left\{G(X(y)): y \in \Omega_{s}\right\}
$$

and that the set of all frameworks $G(q)$ in all Euclidean spaces that are equivalent to $G(\hat{p})$ is given by

$$
\{G(X(y)): y \in \Omega\} .
$$

For more details on set $\Omega$ see [2]. It is clear that framework $G(\hat{p})$ is globally rigid if and only if the set $\Omega_{r}$ is a singleton. Furthermore, $G(\hat{p})$ is universally rigid if and only if the set $\Omega$ is a singleton. Note that $\Omega$ is a closed convex set. This makes the universal rigidity problem amenable to SDP. On the other hand, the global rigidity problem is much harder to tackle since $\Omega_{r}$ is, in general, non-convex due to the rank constraint.

The following technical lemma establishes the connection between the projected Gram matrix of a given framework $G(\hat{p})$ and the Gale matrix of $G(\hat{p})$. 
Lemma 3.1 (Alfakih [3]) Let $G(\hat{p})$ be a given framework with $n$ vertices in $\mathbb{R}^{r}$ for some $r \leq n-2$; and let $Z$ be the Gale matrix of $G(\hat{p})$. Further, let $U$ and $W$ be the matrices whose columns form orthonormal bases of the nullspace and the rangespace of $\hat{X}$, where $\hat{X}$ is the projected Gram matrix associated with $\hat{p}$. Then

1. $V U=Z Q$ for some nonsingular matrix $Q$, i.e., $V U$ is a Gale matrix.

2. $V W=P Q^{\prime}$ for some nonsingular matrix $Q^{\prime}$, where $P$ is the configuration matrix of $\hat{p}$.

\section{Positive Semidefinite Stress Matrices}

Using the results of the previous section, we focus in this section on stress matrices that are positive semidefinite. Consider the following pair of dual SDP problems, where $\hat{X}$ is the projected Gram matrix of a given framework $G(\hat{p})$ in $\mathbb{R}^{r}$, and $M^{i j}$ are as defined in (12).

$$
\begin{array}{ccc}
\text { (P): } \max _{y} & 0^{T} y \\
\text { subject to } & \hat{X}+\sum_{(i, j) \notin E} y_{i j} M^{i j} \succeq 0 . \\
(\mathrm{D}): \quad \operatorname{trace}(\hat{X} Y) & \\
& \min _{Y} \quad & \text { for }(i, j) \notin E, \\
\text { subject to } \quad \operatorname{trace}\left(Y M^{i j}\right)=0 & Y \succeq 0 .
\end{array}
$$

Two remarks concerning problems (17) and (18) are in order here. First, it follows from (13) that the set of all frameworks $G(X(y))$ that are equivalent to $G(\hat{p})$, is equal to the the set of feasible solutions of the primal problem (17). Furthermore, since the objective function of problem (17) is identically equal to zero, the set of all frameworks that are equivalent to $G(\hat{p})$, is also equal to the set of optimal solutions of problem (17).

Second, let $W$ and $U$ be the $(n-1) \times r$ and $(n-1) \times \bar{r}$ matrices whose columns form orthonormal bases for the rangespace and the nullspace of $\hat{X}$ respectively. Since $\hat{X} \succeq 0$, any optimal solution of the dual problem (18) must be of the form $Y=U \Psi U^{T}$ for some $\Psi \succeq 0$. Furthermore, using (12), trace $\left(Y M^{i j}\right)=0$ implies that trace $\left(V U \Psi U^{T} V^{T} E^{i j}\right)=0$. Thus by Lemma 3.1 we have $\left(Z \Psi^{\prime} Z^{T}\right)_{i j}=0$ for all $(i, j) \notin E$, where $\Psi^{\prime}=Q \Psi Q^{T} \succeq 0$, for some nonsingular matrix $Q$. Hence we have the following theorem. 
Theorem 4.1 Let $S$ be a positive semidefinite stress matrix of framework $G(\hat{p})$. Then $Y$ is an optimal solution of the dual problem (18), where

$$
Y=V^{T} S V \text { and } S=V Y V^{T} .
$$

That is, $Y$ is a "projected stress matrix" of $G(\hat{p})$.

The connection between the stress matrix and the optimal dual solutions of certain SDP problems in tensegrity theory was first observed in [21].

A main ingredient in Connelly's proof of the "if" part of Theorem 2.1 is the following theorem.

Theorem 4.2 (Connelly [9]) Let $G(\hat{p})$ be a given framework in $\mathbb{R}^{r}$ and let $S$ be a stress matrix of $G(\hat{p})$. If $G(\hat{p})$ is generic, then $S$ is a stress matrix of any framework $G(q)$ in $\mathbb{R}^{r}$ that is equivalent to $G(\hat{p})$.

A similar result is presented next, where the assumption of the genericness of the framework is replaced by the assumption of positive semi-definiteness of the stress matrix. This result is a simple consequence of SDP complementary slackness.

Theorem 4.3 Let $G(\hat{p})$ be a given framework, generic or otherwise, in $\mathbb{R}^{r}$ and let $S$ be a stress matrix of $G(\hat{p})$. If $S \succeq 0$, then $S$ is a stress matrix of any framework $G(q)$ that is equivalent to $G(\hat{p})$.

Proof. Let $\hat{X}$ and $X$ be the projected Gram matrices of frameworks $G(\hat{p})$ and $G(q)$ respectively. It suffices to show that $X Y=0$, where $Y=V^{T} S V$. But from (17) we have that $X Y=\hat{X} Y+\sum_{(i, j) \notin E} y_{i j} M^{i j} Y=\sum_{(i, j) \notin E} y_{i j} M^{i j} Y$. Thus it follows from (18) that trace $(X Y)=0$. Hence $X Y=0$ since both $X \succeq 0$ and $Y \succeq 0$.

Next we characterize frameworks that admit a non-zero positive semidefinite stress matrix.

\section{Frameworks with Positive Semidefinite Stress Matri- ces}

In this section, we use the following known semidefinite version of Farkas lemma to characterize frameworks that admit a non-zero positive semidefinite stress matrix. A proof is added for completeness. 
Lemma 5.1 Let $A^{0}, A^{1}, A^{2}, \ldots, A^{k}$ be given symmetric matrices of order $n$. Then exactly one of the following two statements hold.

1. $\exists y \in \mathbb{R}^{k}$ such that $A^{0}+\sum_{i=1}^{k} y_{i} A^{i} \succ 0$,

2. $\exists Y \succeq 0, Y \neq 0$, trace $\left(Y A^{0}\right) \leq 0$ and trace $\left(Y A^{i}\right)=0$ for $i=1, \ldots, k$.

Proof. First we prove that Statements 1 and 2 can not hold at the same time. Assume that there exist $y \in \mathbb{R}^{k}$ and $Y \succeq 0, Y \neq 0$ such that $A^{0}+\sum_{i=1}^{k} y_{i} A^{i} \succ 0$, trace $\left(Y A^{0}\right) \leq 0$, and trace $\left(Y A^{i}\right)=0$ for $i=1, \ldots, k$. Then $0<\operatorname{trace}\left(Y\left(A^{0}+\right.\right.$ $\left.\left.\sum_{i=1}^{k} y_{i} A^{i}\right)\right)=\operatorname{trace}\left(Y A^{0}\right) \leq 0$, a contradiction.

Now assume that Statement 1 does not hold and let $\mathcal{L}=\left\{C \in \mathcal{S}_{n}: C=\right.$ $A^{0}+\sum_{i=1}^{k} y_{i} A^{i}$ for some $\left.y \in \mathbb{R}^{k}\right\}$. Then $\mathcal{L} \cap\{C: C \succ 0\}=\emptyset$. By the separation theorem [19], there exists a $Y \in \mathcal{S}_{n}, Y \neq 0$ such that trace $(Y C) \geq 0$ for all $C \succ 0$, which implies that $Y \succeq 0$; and trace $(Y C) \leq 0$ for all $C \in \mathcal{L}$, which implies that trace $\left(Y A^{i}\right)=0$ for $i=1, \ldots, k$ and trace $\left(Y A^{0}\right) \leq 0$. Therefore, Statement 2 holds.

Before we present our characterization, in the following theorem, of frameworks that admit a non-zero positive semidefinite stress matrix, note that a framework of $n$ vertices in $\mathbb{R}^{n-1}$ is a framework where its vertices are located at affinely independent points.

Theorem 5.1 Let $G(\hat{p})$ be a given framework with $n$ vertices in $\mathbb{R}^{r}$. Then $G(\hat{p})$ admits a non-zero positive semidefinite stress matrix $S$ if and only if there does not exist a framework $G(q)$ in $\mathbb{R}^{n-1}$ that is equivalent to $G(\hat{p})$.

Proof. Let $\hat{X}$ be the projected Gram matrix of framework $G(\hat{p})$ and let $U$ be the matrix whose columns form an orthonormal basis for nullspace of $\hat{X}$. Then there does not exist a framework $G(q)$ in $\mathbb{R}^{n-1}$ that is equivalent to $G(\hat{p})$ if and only if there does not exist a $y \in \mathbb{R}^{\bar{m}}$ such that $X(y)=\hat{X}+\sum_{(i, j) \notin E} y_{i j} M^{i j} \succ 0$, if and only if there exists a $Y \succeq 0, Y \neq 0$, trace $(Y \hat{X}) \leq 0$ and trace $\left(Y M^{i j}\right)=0$ for all $(i, j) \notin E$. The first equivalence follows from (15) since rank $X(y)=n-1$ if and only if $X(y) \succ 0$. The second equivalence follows from Lemma 5.1. Now since $\hat{X} \succeq 0$, it follows that trace $(Y \hat{X}) \leq 0$ is equivalent to trace $(Y \hat{X})=0$. It also follows that $Y=U \Psi U^{T}$ for some positive semidefinite matrix $\Psi$.

Therefore, there does not exist a framework $G(q)$ in $\mathbb{R}^{n-1}$ that is equivalent to $G(\hat{p})$ if and only if there exists a non-zero positive semidefinite matrix $\Psi$ such that trace $\left(U \Psi U^{T} M^{i j}\right)=0$ for all $(i, j) \notin E$. But, it follows from the definition of $M^{i j}$ in (12) and from Lemma 3.1 that $-2\left(\operatorname{trace}\left(U \Psi U^{T} M^{i j}\right)\right)=\operatorname{trace}\left(U \Psi U^{T} V^{T} E^{i j} V\right)$ $=$ trace $\left(Z \Psi^{\prime} Z^{T} E^{i j}\right)$ where $\Psi^{\prime}=Q \Psi Q^{T}$ for some nonsingular matrix $Q$. The result 
follows from Lemma 2.1 since the matrix $Z \Psi^{\prime} Z^{T}$ whose $i j$ th entries vanish for all $(i, j) \notin E$ is a stress matrix of $G(\hat{p})$ and since $\Psi^{\prime}$ is nonzero positive semidefinite if and only if $\Psi$ is nonzero positive semidefinite.

\section{A Sufficient Condition for Universal Rigidity}

We apply the notion of SDP non-degeneracy to the pair of dual problems (17) and (18) to obtain a sufficient condition for universal rigidity of frameworks. We also show that Condition (6) in Theorem 2.2 follows from this sufficient condition when the given framework is generic.

Let $S$ be a given positive semidefinite stress matrix of rank $s$ of framework $G(\hat{p})$. Let $W^{\prime}$ and $U^{\prime}$ be the $(n-1) \times s$ and $(n-1) \times(n-1-s)$ matrices whose columns form orthonormal bases for the rangespace and the nullspace of $Y=V^{T} S V$ respectively. Following [6], let

$$
\mathcal{L}=\operatorname{span}\left\{M^{i j}:(i, j) \notin E\right\}
$$

and let

$$
\mathcal{T}_{Y}=\left\{C \in \mathcal{S}_{n-1}: C=\left[\begin{array}{ll}
W^{\prime} & U^{\prime}
\end{array}\right]\left[\begin{array}{cc}
\Phi_{1} & \Phi_{2} \\
\Phi_{2}^{T} & 0
\end{array}\right]\left[\begin{array}{c}
W^{\prime T} \\
U^{\prime T}
\end{array}\right]\right\},
$$

where $\Phi_{1}$ is a symmetric matrix of order $s$ and $\Phi_{2}$ is $s \times(n-1-s)$. $\mathcal{T}_{Y}$ is the tangent space, at $Y$, to the set of $(n-1) \times(n-1)$ symmetric matrices of rank $s$.

Definition 6.1 (Alizadeh et al [6]) $\hat{Y}$ is said to be non-degenerate if

$$
\mathcal{T}_{Y}+\mathcal{L}^{\perp}=\mathcal{S}_{n-1},
$$

otherwise, $Y$ is called degenerate.

Equation (20) is equivalent to

$$
\mathcal{T}_{Y}^{\perp} \cap \mathcal{L}=\{0\}
$$

where $\mathcal{T}_{Y}^{\perp}$ is the orthogonal complement of $\mathcal{T}_{Y}$, namely

$$
\mathcal{T}_{Y}^{\perp}=\left\{C \in \mathcal{S}_{n-1}: C=U^{\prime} \Phi U^{\prime T}\right\} .
$$

The following theorem is well known. 
Theorem 6.1 (Alizadeh et al [6]) Consider a pair of dual SDP problems, where $A^{0}, A^{1}, \ldots, A^{k}$ are given matrices in $\mathcal{S}_{n-1}$, and $b$ is a given vector in $\mathbb{R}^{k}$.

$$
\begin{gathered}
(P): \quad \max _{y} \quad b^{T} y \\
\text { subject to } \quad A^{0}-\sum_{i=1}^{k} y_{i} A^{i} \succeq 0 . \\
(D): \quad \min _{Y} \quad \operatorname{trace}\left(A^{0} Y\right) \\
\text { subject to } \quad \text { trace }\left(Y A^{i}\right)=b_{i} \quad \text { for } i=1, \ldots, k, \\
Y \succeq 0 .
\end{gathered}
$$

If the dual problem has a non-degenerate optimal solution, then the primal optimal solution is unique.

Applying this theorem to the pair of dual problems (17) and (18) we get the following theorem.

Theorem 6.2 Given a framework $G(\hat{p})$ in $\mathbb{R}^{r}$, let $S$ be a positive semidefinite stress matrix of $G(\hat{p})$ and let $U^{\prime}$ be the matrix whose columns form an orthonormal basis of $Y=V^{T} S V$. If the trivial solution, $\Phi=0$ and $y_{i j}=0$ for all $(i, j) \notin E$, is the only solution of the system of equations:

$$
U^{\prime} \Phi U^{\prime T}+\sum_{(i, j) \notin E} y_{i j} M^{i j}=0 .
$$

Then $G(\hat{p})$ is universally rigid.

Proof. Assume that the only solution of (22) is the trivial solution. Then $\mathcal{T}_{Y}^{\perp} \cap \mathcal{L}=\{0\}$. Hence, $Y$ is a non-degenerate optimal solution of (dual) problem (18). Thus, the set of optimal solution of (primal) problem (17) is a singleton. Therefore, $G(\hat{p})$ is universally rigid.

Two remarks concerning (22) are in order here. First, $\Phi=0$ if and only if $y_{i j}=0$ for all $(i, j) \notin E$ since the set $\left\{M^{i j}:(i, j) \notin E\right\}$ is linearly independent, and since $U^{\prime}$ is full column rank. Second, the condition that the only solution of (22) is the trivial solution can be equivalently stated as the condition that a set of certain matrices is linearly independent.

Next we focus our attention on generic frameworks. The following lemma is needed in the proof of Theorem 6.3 below. 
Lemma 6.1 (Alfakih [4]) Let $G(p)$ be a generic framework in $\mathbb{R}^{r}$ and let each vertex of $G$ have a degree at least $r$. Further, let $Z$ be the Gale matrix of $G(p)$. Then there does not exist a non-zero $y=\left(y_{i j}\right) \in \mathbb{R}^{\bar{m}}$ such that

$$
\sum_{(i, j) \notin E} y_{i j} V^{T} E^{i j} Z=0 .
$$

Theorem 6.3 Theorem 2.2 follows as a corollary of Theorem 6.2 .

Proof. Assume that framework $G(p)$ in $\mathbb{R}^{r}$ is generic and that $S=Z \Psi Z^{T}$ is a stress matrix of $G(p)$ where matrix $\Psi$ is $\bar{r} \times \bar{r}$ positive definite (recall that $\bar{r}=n-1-r)$. We will show that, in this case, the only solution of $(22)$ is the trivial solution. Hence it would follow from Theorem 6.2 that $G(p)$ is universally rigid.

First, we show that every vertex of $G$ has a degree at least $r+1$. For assume, to the contrary, that the degree of one node of $G$, say node 1 , is $\leq r$, and wlog assume that nodes $2,3, \ldots, \bar{r}+1$ are not adjacent to node 1 . Thus it follows from Lemma 2.2 that $z^{2}, z^{3}, \ldots, z^{\bar{r}+1}$ form a basis in $\mathbb{R}^{\bar{r}}$. Hence there exist $\lambda_{2}, \lambda_{3}, \ldots, \lambda_{\bar{r}+1}$, not all of which are zeros, such that $z^{1}=\lambda_{2} z^{2}+\lambda_{3} z^{3}+\cdots+\lambda_{\bar{r}+1} z^{\bar{r}+1}$. Therefore, $z^{1^{T}} \Psi z^{i}=0$ for $i=2, \ldots, \bar{r}+1$ implies that $z^{1} \Psi z^{1}=0$ and therefore, $\Psi$ is singular contradicting our assumption that $\Psi \succ 0$.

Now, the stress matrix $S=Z \Psi Z^{T}$ with $\Psi \succ 0$ also implies that the columns of the matrix $\left[\begin{array}{ll}P & e\end{array}\right]$ form a basis for the nullspace of $S$ since in this case, rank $S=n-1-r$. Consequently, it follows from Lemma 3.1 that the columns of $V^{T} P$ form a basis for the nullspace of $Y=V^{T} S V$. Thus, in this case (22) reduces to

$$
V^{T}\left(P \Phi^{\prime} P^{T}+\sum_{(i, j) \notin E} y_{i j}^{\prime} E^{i j}\right) V=0 .
$$

But (23) is equivalent to

$$
P \Phi^{\prime} P^{T}+\sum_{(i, j) \notin E} y_{i j}^{\prime} E^{i j}=a e^{T}+e a^{T}
$$

for some $n$-vector $a$.

Thus it suffices to show that the only solution of (24) is the trivial solution.

Assume, to the contrary, that $(24)$ has a solution $\Phi^{\prime} \neq 0, y^{\prime}=\left(y_{i j}^{\prime}\right) \neq 0$. Then by multiplying (24) from the left by $V^{T}$ and from the right by $Z$ we get $\sum_{(i, j) \notin E} y_{i j}^{\prime} V^{T} E^{i j} Z=0$. But this contradicts, from Lemma 6.1, our assumption that $G(p)$ is generic. Thus the result follows.

Finally, we end with the following numerical example that illustrates some of the results of this paper. 


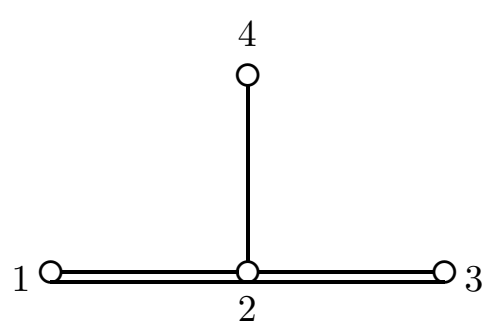

Figure 3: The non-generic framework $G(p)$ in $\mathbb{R}^{2}$ of Example 6.1. Here, the missing edges of $G$ are $(4,1)$ and $(4,3)$.

Example 6.1 Consider the non-generic framework $G(p)$ in $\mathbb{R}^{2}$ depicted in Figure 3. $G(p)$ has two missing edges $(4,1)$ and $(4,3)$, and $\bar{r}=n-1-r=1$ in this case. It is easy to show that the Gale matrix $Z$ and a stress matrix $S$ of $G(p)$ are

$$
Z=\left[\begin{array}{llll}
1 & -2 & 1 & 0
\end{array}\right]^{T} \text { and } S=Z Z^{T} .
$$

Note that $S$ is positive semidefinite with rank $1=\bar{r}$. Thus $G(p)$ satisfies Condition (6) of Theorem 2.2. However, $G(p)$ is obviously not universally rigid (in fact it is not even globally rigid). This shows that the assumption in Theorem 2.2 that the framework is generic can not be dropped.

On the other hand, since stress matrix $S$ is non-zero positive semidefinite, it follows from Theorem 5.1 that there does not exist a framework in $\mathbb{R}^{3}$ that is equivalent to $G(p)$. Indeed, this is obviously the case. In fact, $G(p)$ has an infinite number of equivalent frameworks in $\mathbb{R}^{2}$, and it has two equivalent frameworks in $\mathbb{R}^{1}$ : One where node 4 coincides with node 1, and one where node 4 coincides with node 3.

\section{Summary and Concluding Remarks}

In this paper, we used semidefinite programming techniques to tackle the universal rigidity problem. This problem was made amenable to SDP by the use of projected Gram matrices to represent point configurations in Euclidean space, and by the characterization of the set of all frameworks that are equivalent to a given framework in $\mathbb{R}^{r}$, in terms of a convex closed set formed by the intersection of the positive semidefinite cone with an affine subspace.

We characterized frameworks that admit non-zero positive semidefinite stress matrices, and we obtained some new results concerning such matrices. We used the notion of semidefinite programming non-degeneracy to obtain a sufficient condition for universal rigidity, and we showed that this condition yields the known sufficient condition for generic universal rigidity.

The fact that the set of all frameworks in $\mathbb{R}^{r}$ that are equivalent to a given framework in $\mathbb{R}^{r}$ is non-convex, due to the rank constraint, makes the global rigidity 
problem much harder to tackle. This also provides a challenge to extend the techniques used in the paper to global rigidity and to indefinite stress matrices. Perhaps a first step in that direction would be to use Moreau Theorem [17] to express an indefinite stress matrix as the difference between two orthogonal positive semidefinite matrices.

\section{References}

[1] A. Y. Alfakih. Graph rigidity via Euclidean distance matrices. Linear Algebra Appl., 310:149-165, 2000.

[2] A. Y. Alfakih. On the set of realizations of edge-weighted graphs in Euclidean spaces, 2005. Research Report, Mathematics and Statistics, University of Windsor.

[3] A. Y. Alfakih. On dimensional rigidity of bar-and-joint frameworks. Discrete Appl. Math., 155:1244-1253, 2007.

[4] A. Y. Alfakih. On the universal rigidity of generic bar frameworks, 2007. submitted.

[5] A. Y. Alfakih, A. Khandani, and H. Wolkowicz. Solving Euclidean distance matrix completion problems via semidefinite programming. Comput. Optim. Appl., 12:13-30, 1999.

[6] F. Alizadeh, J. A. Haeberly, and M. L. Overton. Complementarity and nondegeneracy in semidefinite programming. Math. Programming, Ser. B, 77:111$128,1997$.

[7] P. Biswas and Y. Ye. Semidefinite programming for ad hoc wireless sensor network localization. Technical report, Dept. of Management Science and Engineering, Stanford University, 2003.

[8] R. Connelly. Unpublished manuscript.

[9] R. Connelly. Generic global rigidity. Discrete Comput. Geom., 33:549-563, 2005.

[10] T.F. Cox and M.A. Cox. Multidimensional scaling. Chapman and Hall, 2001.

[11] G. M. Crippen and T. F. Havel. Distance Geometry and Molecular Conformation. Wiley, New York, 1988. 
[12] Y. Ding, N. Krislock, J. Qian, and H. Wolkowicz. Sensor network localization, euclidean distance matrix completions, and graph realization, 2006. CORR 2006-23, Dept of Combinatorics and Optimization, U. of Waterloo.

[13] T. Eren, D.K. Goldenberg, W. Whiteley, Y.R. Yang, A.S. Morse, B.D.O. Anderson, and P.N. Belhumeur. Rigidity, computation, and randomization in network localization, 2004. IEEE INFOCOM.

[14] D. Gale. Neighboring vertices on a convex polyhedron. In Linear inequalities and related system, pages 255-263. Princeton University Press, 1956.

[15] S. J. Gortler, A. D. Healy, and D. P. Thurston. Characterizing generic global rigidity, 2007. arXiv/0710.0926.

[16] J. De Leeuw and W. Heiser. Theory of multidimensional scaling. In P. R. Krishnaiah and L. N. Kanal, editors, Handbook of Statistics, volume 2, pages 285-316. North-Holland, 1982.

[17] J. J. Moreau. Décomposition orthogonale d'un espace hilbertien selon deux cônes mutuellement polaires. C. R. Acad. Sci Paris, 255:238-240, 1962.

[18] T.K. Pong and P. Tseng. Robust edge-based semidefinite programming relaxation of sensor network localization. Technical report, U. of Washington, 2009.

[19] R. T. Rockafellar. Convex analysis. Princeton University Press, 1970.

[20] J. B. Saxe. Embeddability of weighted graphs in k-space is strongly NP-hard. Proc. 17th Allerton Conf. in Communications, Control, and Computing, pages 480-489, 1979.

[21] A. M-C So and Y. Ye. A semidefinite programming approach to tensegrity theory and realizability of graphs. In 17th annual ACM-SIAM symposium on discrete algorithms, pages 766-775, 2006.

[22] A. M-C So and Y. Ye. Theory of semidefinite programming for sensor network localization. Math. Program. Ser. B, pages 367-384, 2007.

[23] Z. Wang, S. Zheng, Y. Ye, and S. Boyd. Further relaxations of the semidefinite programming approach to sensor network localization. SIAM J. Optim., pages 655-673, 2008. 